\title{
China's Current Social Conflicts and Governance
}

\author{
Yi Liu \\ Southwest Jiaotong University, Chengdu city, Sichuan province, China \\ ly741388439@sina.com
}

\begin{abstract}
Keywords: Social conflict; Social conflict theory; Social psychology; Causes of social conflict; Conflict governance
\end{abstract}

\begin{abstract}
Our study concentrates on China's current social conflicts, the analysis focuses on the reasons for its existence and features, on this basis, explores the patterns and methods of conflict governance. We adopt literature research methodology to complete this essay. Underlying theoretical studies of conflicts at home and abroad, we must combine with the country's actual conditions, viewing specifically and dialectically on social conflicts in China. Under Chinese characteristic socialism system, we try to understand what are some basic and specific characters of social conflicts that happen in China, and what are expressions of social conflicts. Meanwhile, we aim at exploring the rooted multilevel causes leading to conflict occurrence.During the process of studying social conflicts, combining various disciplines to find effective means of governance is important.
\end{abstract}

\section{Introduction}

Nowadays China is in the period of transition of socialist society. This indicates that the current China is in the period of the transition from the old to the new, the social conflict in this process has distinct characteristics of the times. The modernization of China, the rapid development of information technology, the gradual expansion of the global vision, all of these also make social conflicts have a new and diverse mobilization and expression ${ }^{[1]}$.

The present studies focuse on the structural analysis of domestic social conflicts, in which the political and economic level is dominant with less systematic theory, and the domestic researches on conflict governance are relatively weak, and have not formed the unique theoretical system. This paper aims at comprehensively and deeply analyzing the causes of social conflicts, summarizing the characteristics and different manifestations of social conflicts, exploring innovative governance models, and innovating the governance perspective of social conflicts.

\section{Social Conflict Classification}

In the light of the prophase studies on social conflict classification in our country, social conflict will be divided into antagonistic and nonantagonistic conflict, that is, non constructive and constructive conflict, the standard of division is mainly based on whether the contradiction of social conflict can be reconciled.Nonantagonistic conflicts, to some extent, are social vents, which play a positive role in society and can constructively promote social transformation.Combined with China's current situation, the outbreak of the conflicts among the parties can be finally settled through mediation and other forms, so the social conflicts can be mostly attributed to this category.

\section{Trends in Social Conflict}

Variety of Manifestation. The traditional social conflict in China mainly refers to the conflict of direct interest demands, however,with the deepening of the process of modernization,social conflicts, which have more characteristics of the times and richer forms of expression, reflect more indirect interests, such as social conflicts related to ecological problems, religious conflicts, network ideological conflicts and labor conflicts.

Conflicts to Vent People's Anger. The presence of the sense of relative deprivation leads to the 
happening of conflicts that can just show people's anger. In order to vent the anger, such conflicts emerged, and gradually become outstanding in recent years. It's interesting that the masses have no direct relationship with the original events, and they can't immediately benefit from such events either,people just want to give full vent to their accumulated discontent by taking part in these events. This is the most direct purpose of such conflicts, and is also the most intuitive expression of vulnerable people.

\section{Causes of Social Conflict}

Economic Reasons. Most of the domestic analyses of the causes of social conflict emphasize that economic factors are the root cause ${ }^{[2]}$. It brings all sorts of contradictions from the source and then sharpen them inevitably. In a society, all kinds of external social resources are always finite.Population pressure,coupled with uneven regional development, made it difficult to ensure that everyone can enjoy the same social resources ${ }^{[3]}$.This situation allows vested interests of all parties and those who have failed to game and struggle constantly for their own interests. The outcome of this conflict of interest will, in turn, reinforce the economic imbalance.

Political Reasons. The reform of the political system has not yet achieved remarkable results, to a certain extent, this makes democracy and the rule of law have great limitations and it's also clear that these systems can't be fundamentally developed and improved.Because of the restrictions of the laws and regulations and the disadvantages of the channels of communication and participation, people cannot express their political demands in time or take part in political affairs effectively,this gap feeling has made people lose confidence in the government,it means that the credibility and legitimacy of the government has been challenged.Under the oppressive political circumstances, our country gets stuck in the dilemma of democratization, this is also an important cause of the occurrence of social conflicts.

Religious Reasons. This paper mainly discusses the stimulation of religious culture. So far social conflicts that happen in China have partly proved the great influence of religion.Religious culture has the absolute authority over the national members in the field of ideology, it represents tradition and obedience, once it becomes a political or economic tool, a single spark can start a prairie fire.The difference of religious beliefs of all nationalities and the difference of cultural accumulation often bring the deepest impetus to social conflicts at first, and they are also the underlying causes of conflicts ${ }^{[4]}$.

Moral Reasons. Nowadays social morality is weaker in binding people than bofore, because of the decay of morals, it's difficult for people in authority to resist the various temptations brought by the rapid economic development,and this means the breeding of corruption and the abuse of power.The influence of corruption is indirect and far-reaching, which means that rules and regulations are losing their proper effect due to the destruction of procedure and institutionalization.Under this unhealthy ways and customs, people can not defend their own interests through normal means. The existence of corruption makes social order confused,under the chaotic social order, social transformation will be conducted at great cost,social conflicts are inevitable.

\section{Analysis on Countermeasures of Social Conflict Governance}

Social conflict is not a confrontation that cannot be reconciled, nor is it a so-called zero-sum game,social conflict should be more of a relationship ${ }^{[5]}$,so it's important for us to discard the "total fixation" thinking hypothesis and seek a win-win integrated solution which has high feasibility.This is the correct direction and target of governance in the face of the social conflicts that have taken place. Howbeit, the governance of social conflicts is not merely the resolution of conflicts,the prevention and control of social conflict is also the main content of conflict governance $^{[6]}$.In the process of social conflict governance, it is more important to grasp the accurate and reasonable governance principles and implement appropriate and effective governance strategies. 
Multi-agent Governance. In the light of Ostrom's polycentric governance theory, we should adhere to the "multi-agent governance" in the process of governing social conflicts, abandon the idea that the government is the only actor during the process of governance, and include social organizations and the public into the main body of governance. The government must think highly of the cooperative governance with the third parties, and pay attention to building a stable community structure to deepen the transverse links between the two sides of the conflict and to avoid social conflicts escalating. In our country, the multi-agent governance will still be dominated by the government,however, the ultima settlement of the conflict requires the coordination and cooperation between the government and the third parties. In this process ,the status and function of the three parties such as social organizations must be aggrandized, otherwise, it will be difficult for them to become the pillar of conflict governance.

Governance by Law. Good governance should go hand in hand with the rule of law, the social conflict governance in China must comply with the law , abide by the law and give full play to the social control function of the law.Governing by operation of law is to put everything under the protection of norms ${ }^{[7]}$, which means institutionalization and routinization and that disorder and confusion will be averted on purpose. First of all ,governing according to law aims at the main body of governance,because they are the main observers of laws, regulations and other norms in the process of social conflict governance. As one of the main bodies of governance, our government should publicize indispensable legal knowledge,make people know the laws and abide by its rules so that they can express their interest demands legitimately. Appealing to the law will clear up many potential remote causes of conflicts.

Source Control. The current social conflict governance tends to focus on the ex post emergency management, while source control should be of paramount importance. The supplementary means of source control is to establish a social early warning mechanism with operational index system and model $^{[8]}$. The early warning and assessment of the risks of social conflicts that are about to happen will help us to do prevention and control work to the maximum extent. The best effect of source control is to nip conflicts in the bud by adopting various governance measures, nevertheless, when the state of affairs expand inevitably, with the aid of early warning mechanism, it can still help us to find the appropriate solution as soon as possible, turn the passive situation into active situation.

\section{Countermeasure Proposal}

According to the characteristics and trends of social conflicts during the transitional period, the countermeasures can be divided into the following parts:

Improvement of Interest Demands Mechanisms. The rapid increase in the number of social conflict events is closely related to the process of urbanization, ultimately, social conflict is still a dispute over interest. In the face of such social conflicts, our government should not only improve the relevant policies and regulations and make sure that they transparent ,but also fully respect the interest demands of the masses,all the work should be performed with negotiation instead of enforcement.Throughout the process,people aought to be given full participation and discourse right,and their reasonable demands should be satisfied as soon as possible, but beyond that,it's the legality and openness of the work process that the government should ensure.

We should improve the mechanism of interests demands, the social organization should also be included in the innovation of the interests demand mechanism while improving the existing mechanism of demands at the same time,so that the channels of public interests expression can be more extensive. This requires the improvement of the collaborative governance ability of the government and social organizations, and it also demands that social organizations should strengthen their influence.As the coming of the information age, the masses can also use the influence of the new media to augment the breadth and intensity of their expressions, so that their reasonable demands can be solved in a timely manner.

Establish Nimby Communication Mechanism. Although the manifestations of social conflicts are diverse,the The proportion of environmental social conflict and labor conflict is relatively 
obvious.

The deep-seated governance of environmental collision is to adhere to sustainable development, improve the environmental conditions, and enhance the quality of life of residents at its roots, which implies the Optimization of industrial structure and the transformation of economic development patterns. In addition to the protection of the ecological environment, establishing the communication mechanism of Nimby(not in my backyard) facilities is much more direct.Communicating with the public in advance about the location problems and the follow-up problems that may arise after construction, so as to eliminate the information asymmetry, will be the advisable choice of the government.

Confronted with labor conflict,depending merely on communication is not enough, people should also rely on the social mediation mechanism when the communication is poor and the interests of both sides cannot be maximized. At present, China has even promulgated relevant laws to help establish and improve the major mediation mechanism,that is, people's mediation, judicial mediation and administrative mediation, the common forms of mediation are administrative appeal, administrative review, labor arbitration and collective bargaining.

Strengthen the Dredging of Conflict Information. Take the cyber ideological conflict as an example, Rumors and other false news spread more quickly by the aid of new media such as the Internet. Government and other social organizations should release authoritative information in time to achieve flexible and accurate information control and guidance, so as to prevent people from being incited by false information.refuting a rumour and disclosing information are very effective to control the scale of the conflicts.Government and other governance entities can use their influence to grasp the true and accurate relevant information promptly and quickly in the initial stage of social conflicts, their ability of dredging information is tested in this process, it's essential for them to do all they can to gain the initiative and resolve the conflicts reasonably.

Pay Attention to Social Mentality Management. The management of social mentality is divided into two aspects, one is the outlet of emotions, the other is the inhibition of bad attitude at its roots.If the governance subject can solve this kind of fuse events duly and reasonably, put forward the solution that satisfy the general public, it will greatly reduce the conflict that vent people's anger, avoid large-scale social conflicts caused by sudden escalations of events. But this is not a key resolvent of social conflicts, although it can control social conflicts, in fact the negative emotions of the masses are still not clear and this way can only be used as a means of emergency management.

Social mentality is mainly based on the sense of fairness, security, participation and trust, the emergence of the bad social mentality of the masses means a series of problems, such as lack of fairness, security risk, insufficient participation and crisis of confidence, under the circumstances, a sense of relative deprivation (mainly fellow deprivation) ${ }^{[9]}$ and accumulated discontent comes into being, which signifies that the government have to make long-term insistent efforts to eliminate the resentment of the masses.For our current government,it's urgent to strengthen the governance capability, transform the concept of governing and build a service-oriented government, only by taking the interests of the masses as the first priority and repairing corruption energetically can our government let the people's confidence and trust in its ruling finally increase. During this process, it's necessary to follow out the "People-oriented" wisdom. In the next place, it is exigent to set up a even fairer and more reasonable income distribution system, establish a fair judicial system $^{[10]}$, and defend social equity and justice. At the same time, people should be encouraged to take part in the political affairs to enhance their sense of political participation and presence.The government should attach importance to the constructive suggestions of the masses, reinforce the communication and interchange, and keep the communication channels unimpeded on the basis of comprehending the psychology, ideas, and demands of the common people.On this foundation, with these multiple approaches, social psychology management can achieve the anticipative results. 


\section{Conclusions and Future Work}

In recent years, with the characteristics of the transition period becoming more obvious, the studies of social conflicts in China are mainly at the political, economic and moral level and they explore the causes of conflicts and propose solutions mostly on the mainstream culture and personal level.The discussions of conflict management are one-sided, and most of them haven't been converted to the view of governance. There is still plenty of room to discuss how to improve the governance model of social conflict.The social conflict governance will be integrated research involving a wide range of subjects, for example,peace psychology and social psychology will provide novel and unique perspectives and methods for the study of governance models of social conflict.More governance subjects will be included,and on this basis, the innovation mechanism of social conflict governance can have a better development in the future.

\section{References}

[1] J. Lakis,Social conflicts and the culture of cooperation in transitional society[J].Baltic Journal of Management,2009,4(2):206-220.

[2] M. Vahabi,.Intergrating social conflict into economic theory[J].Cambridge Journal of Economics, 2010,34(4):687-708.

[3] H. Strulik,Social composition,social conflict and economic development[J].The Economic Journal,2008,118(530):1145-1170.

[4] A.A. Ramiah,M.Hewstone,K.Schmid,Social Identity and Intergroup Conflict[J].Psychological Studies,2011,56(1):44-52.

[5] M. Wieviorka,Social Conflict[J].Current Sociology,2013,61(5-6):696-713.

[6] R.E. Wagner-Pacifici,M. Hall,Resolution of Social Conflict[J].Sociology,2012,38.

[7] L.B. Amsler,The evolution of social norms in conflict resolution[J].Journal of Natural Resources Policy Research,2014,6(4):285-290.

[8] E. MD,Preventing and resolving social conflicts[J].Canadian Mining Journal,2012,133(8):8

[9] L. Mazur,The social psychology of intractable conflicts[J].Culture\&Psychology,2014,20(2):276-281.

[10] G. Mikula,M.Wenzel,Justice and Social Conflict[J].International Journal of Psychology,2000,35:126-135. 